Proceedings of the 2010 Winter Simulation Conference

B. Johansson, S. Jain, J. Montoya-Torres, J. Hugan, and E. Yücesan, eds.

\title{
EVALUATING THE PERFORMANCE OF A COMPLEX POWER AND FREE CONVEYOR SYSTEM IN A FLEXIBLE MANUFACTURING ENVIRONMENT
}

\author{
Ashish Devikar \\ Nikhil Garge \\ Rajesh Welekar \\ Production Modeling India Pvt. Ltd. \\ 112, Milestone Building, Ramdaspeth \\ Nagpur - 440012 INDIA
}

\author{
Karthik Vasudevan \\ Edward Williams \\ Production Modeling Corporation \\ 15726 Michigan Av \\ Dearborn, MI 48126, USA
}

\begin{abstract}
This paper describes the methodology, challenges and findings from a simulation modeling and analysis project dealing with the study of a complex power \& free transportation system in a flexible manufacturing environment. The system under consideration transports six car body types through different stages of the production lifecycle (including body, paint and assembly). As production levels and product mix change, the interactions between different system parameters become too complex to analyze analytically. A simulation study was undertaken to identify bottlenecks and determine/improve the capacity of the $\mathrm{P} \& \mathrm{~F}$ system at peak demand while validating various routing rules at key decision points.
\end{abstract}

\section{INTRODUCTION}

One of the most venerable and still one of the most frequent, uses of simulation in practice is the simulation of manufacturing systems (Miller and Pegden 2000). In this venue, simulation studies can and do aid decision-making to improve efficiency in the face of complexities such as unpredictable downtime occurrences, duration of repair, fluctuation of demand, relative costs of carrying inventory versus stockouts, and complicated routing logic partially dependent on product mix. In any manufacturing context, and especially those wherein the product involved is heavy and bulky (certainly including motor vehicles), material handling and transport costs are a necessary and significant, yet non-value-added expense - a form of muda (Finch 2008). Hence stochastic models, such as discrete-event simulation models, are of major value in examining logistical problems within the "four walls of a facility" (Heragu, Meng, and Zijm 2008). Many contributions to the literature recently have documented the undertaking and value of simulation in improving the performance of material handling and transport systems within a manufacturing context.

For example, (Guasch, Figueras and Fonseca 2009) describe an analysis of a factory railway system used to transport hot steel coils within a steel factory. A study of logistics and transportation problems in a two-story job shop subject to highly seasonal demand is documented in (Farahmand and Balasubramanian 2002). A comprehensive analysis of performance of automatic guided vehicle (AGV) systems supporting logistics within flexible assembly lines appears in (Hoff et al. 1997). Examination of complex networks of conveyors with merging configuration within manufacturing operations is documented in (Jing et al. 1998). In these traditions, the present study documents the simulation and analysis of a power$\&$-free conveyor system, charged with support of highly variable demand levels and complex product mix, in the automotive industry. Applications of discrete-event simulation in manufacturing systems can be classified in two categories: a) Based on the stage of the development of the design of the system. b) Based on the nature of problem to be investigated (Ulgen and Gunal, 1998). 
In this paper, we will discuss the use of simulation techniques to determine bottlenecks in the complex working environment of P\&F system. The P\&F system transported car bodies from 5 different body shops to two paint shops, an intermediate prep shop and then to final assembly lines. Production levels of each body shop vary with shift and this causes severe demand fluctuations. Fluctuation in body shop production not only causes fluctuation in paint and assembly deliveries, but could also impact hanger availability at downstream body shops. Hence a fine balance between pick-ups and drop-offs is essential.

This project was run using the 9 step simulation methodology described in Figure 1. Analysis revealed that there are twin bottlenecks in this system and there are several cases where this causes the classic deadlock situation in a closed loop system. Rules and guidelines to run the P\&F system were refined using the simulation study. It is estimated that using the rules developed from the simulation model cut the need for manual intervention (mainly to resolve deadlock occurrences) by almost $70 \%$ compared to the current setup. The throughput of the system was increases to target levels through iterative analysis.

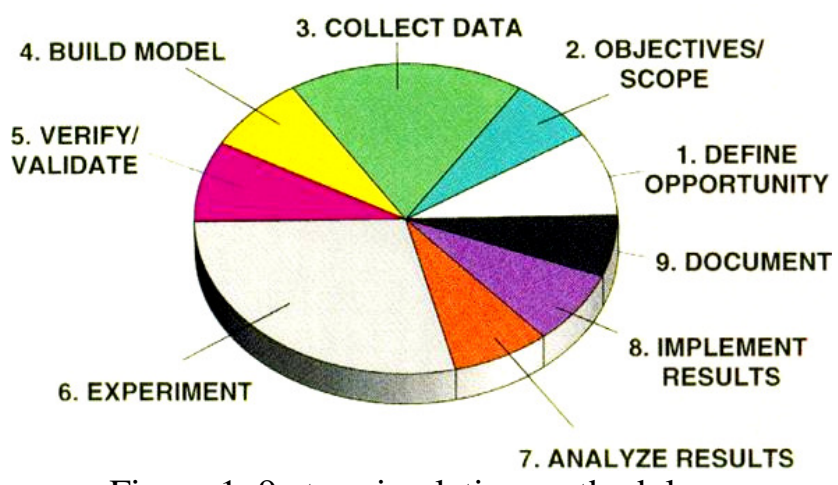

Figure 1: 9-step simulation methodology

\section{SCOPE AND OBJECTIVES}

The automotive manufacturing plant under consideration operates a power $\&$ free $(\mathrm{P} \& \mathrm{~F})$ system that transports car bodies in different stages of production between these different production shops. As the plant was planning to ramp up production and potentially include new models to its portfolio, the manufacturing teams wanted to understand the intricacies of this key material handling system. The core objective of the project was twofold.

- Validate the power \& free system in terms of its capacity to meet current production requirements

- Identify bottlenecks and develop a throughput improvement roadmap to meet future production levels at 652 paint shop deliveries per day

Also, it was hard to analytically predict the magnitude of impact of higher production levels at each of the body shops on the P\&F system. The manufacturing management team needed to develop strategic routing policies to ensure the drop lifts at the body shop were never starved of empty hangers as this would block the body shop production lines and thereby lead to a drop in the throughput. These decision rules had to also adhere to a set of inherent and rigid routing constraints in the P\&F system operation. Additionally optimizing the inventory levels in the storage systems between the each body shop lines and the lift that loaded the P\&F system was also significant both in terms of performance and cost.

\section{OVERVIEW OF THE MANUFACTURING SYSTEM}

A leading automotive manufacturer in India owns and runs a manufacturing plant that produces five models of utility vehicles for the local and international markets. The plant consists of five press \& body shops, two paint shops and multiple final assembly lines. The power and free system used to transport car bodies between different shops had four major functions. 
- Lift bodies in white (BIW i.e. un-painted bodies) from all body shop buffers using drop lifts

- Transport the BIWs to the PTED shop

- Move all bodies that have completed PTED to one of two paint shops

- Deliver painted bodies from each of the paint shops to the respective final assembly line

The power and free conveyor consists of a single main chain on which there are hooks at regular intervals. Each of these hooks can engage a hanger that in turn carries a car body on it. In the current system, there are a total of 215 hangers on the P\&F chain. Hangers can pick up or drop off car bodies using drop lifts that are available at each of the pick-up and drop-off points - i.e. end of each body shop line, start and end of each paint shop and PTED lines. There is also drop lift and at the entry to the assembly line and two drop lifts for delivery to indirect transport that moves some bodies to other plants. Figure 2 shows the high level process flow that is executed using the P\&F system. The P\&F system targets are set as described below.

- Deliver 324 painted vehicles to the final assembly line

- Pickup 300 bodies of Body Type 1 out of the second paint shop

- Deliver 725 vehicles to PTED out of which 73 are taken out to an external plant for assembly

- Deliver 652 vehicles to both the paint shops combined

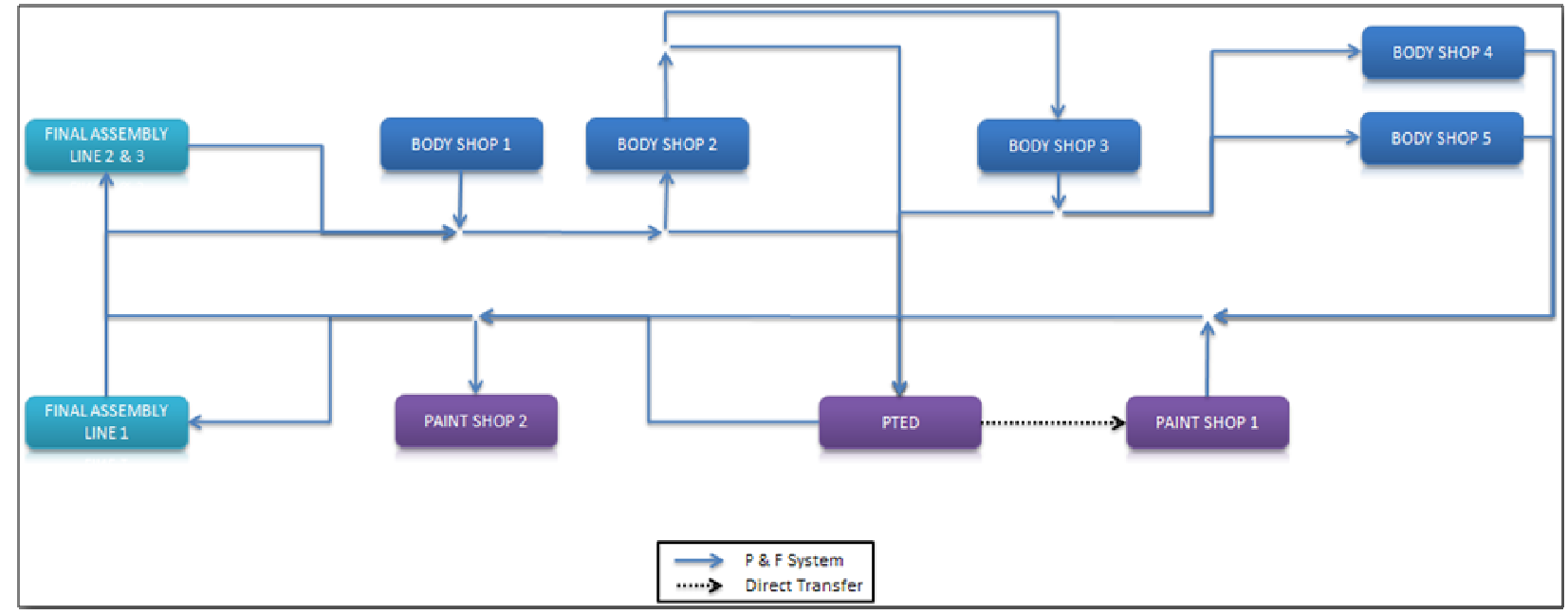

Figure 2: High level process flow diagram

Fig 3 shows a screen shot of the detailed flow diagram, which provides a glimpse of the complexity involved. The target was to achieve complete drops at PTED for the day's production at different body shops and final assembly line (operating in 2 shifts). Fig 4 gives the shift table for the 5 body shops.

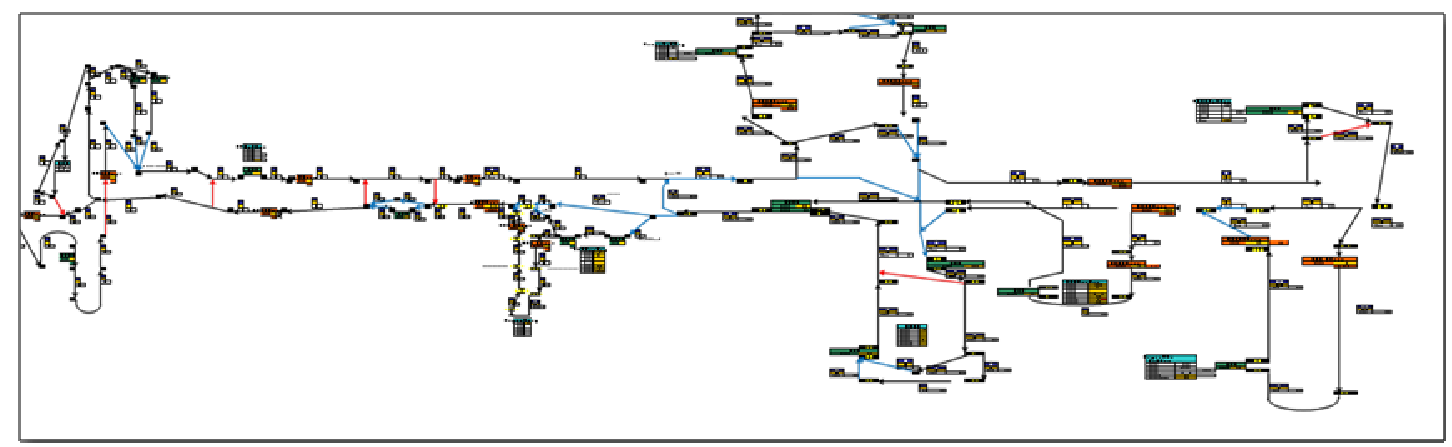

Figure 3: Screen Shot of Detailed Flow Diagram (specifics not shown for confidentiality) 


\begin{tabular}{|l|c|c|c|}
\hline \multicolumn{1}{|c|}{ Shift Table } & I & II & III \\
\hline Bodyshop 1 & On & On & On \\
\hline Bodyshop 2 & On & On & Off \\
\hline Bodyshop 3 & On & On & Off \\
\hline Bodyshop 4 & On & Off & Off \\
\hline Bodyshop 5 & On & Off & Off \\
\hline PTED & On & On & On \\
\hline Paintshop 1 & On & On & Off \\
\hline Paintshop 2 & On & On & On \\
\hline Final Assembly Line 1 & On & On & Off \\
\hline
\end{tabular}

Figure 4: Shift assignment chart

\section{MODELING, VERIFICATION AND VALIDATION}

Picking the right abstraction levels is vital to the success of a simulation project. The biggest challenge for a simulation engineer during the conceptual design phase of the project is modeling at the right abstraction level (Vasudevan et al. 2009). After due research, talking to several stakeholders and completing the analysis of required objectives, the abstraction level of the simulation model was determined. Including all the operational details of every system component would involve too much time and material investment and would not justify ROI for this simulation model. A conceptual model was developed that abstracted system details to a certain level maintaining all key details that are expected to impact key performance indicators.

The scope of modeling was defined as the areas between the body shop's end-of-line buffers and included several system components going up to the drop lift that served final assembly. Critical aspects of operation such as shift patterns and drop/lift requirements at each body shop, paint shop and final assembly was considered in the scope of study. Several system constraints including capacities, rigid safety rules etc were also included in scope. The objective of the study was considered at every phase of the modeling effort to ensure that project time was not spent on modeling unnecessary detail.

A SIMUL8 simulation model was developed that modeled several system components including:

- P\&F sections with accumulation, hangers, slopes and speed variations

- Five body shops along with end of line buffers, cycle time variation and downtimes

- The pre-treatment (PTED) line and two paint shops along with start and end of line buffers with cycle times and downtimes

- Assembly line and transfer locations with cycle times and downtimes

- An automatic body storage system (in front of the assembly line)

- Twelve drop lifts with cycle times and downtimes

Several key data inputs were collected to feed to model:

- Product Mix

- Daily production volumes

- Buffer location and sizes

- Routings / Flow logic / Operating practices

- TAKT times

- Shift hours

- Availability Data

- P\&F segment wise capacity, Number of hangers in system

- Special Rules for routing in P\&F system 


\section{Devikar, Garge, Vasudevan, Welekar and Williams}

The input data was organized in Microsoft Excel ${ }^{\circledR}$ spreadsheet to provide transparency and to facilitate what-if experiments along with ease in handling input changes. Using the Simul8® viewer tool and the Excel ${ }^{\circledR}$ input sheet, even non-simulation experts can conduct experiments and analysis without having to delve into the model.

Several simul8 constructs were used to build the model. These included 'Work stations', 'Resources', 'Conveyors', 'Spreadsheets', 'Variables' and 'Labels'. In order to restrict/gate capacities of different sections of the system the model used 'Groups'. Power and free sections were built using a combination of machines and conveyors. This combination of elements was then used as a module which was repeated across the model to create the holistic system. Development of the model in re-usable modules cut development time significantly. Figure 5 below shows a screen shot of the completed model. Notice that the power and free system has been divided into segments for easy on-screen readability. The model also includes a dashboard to view key performance indicators as it runs.

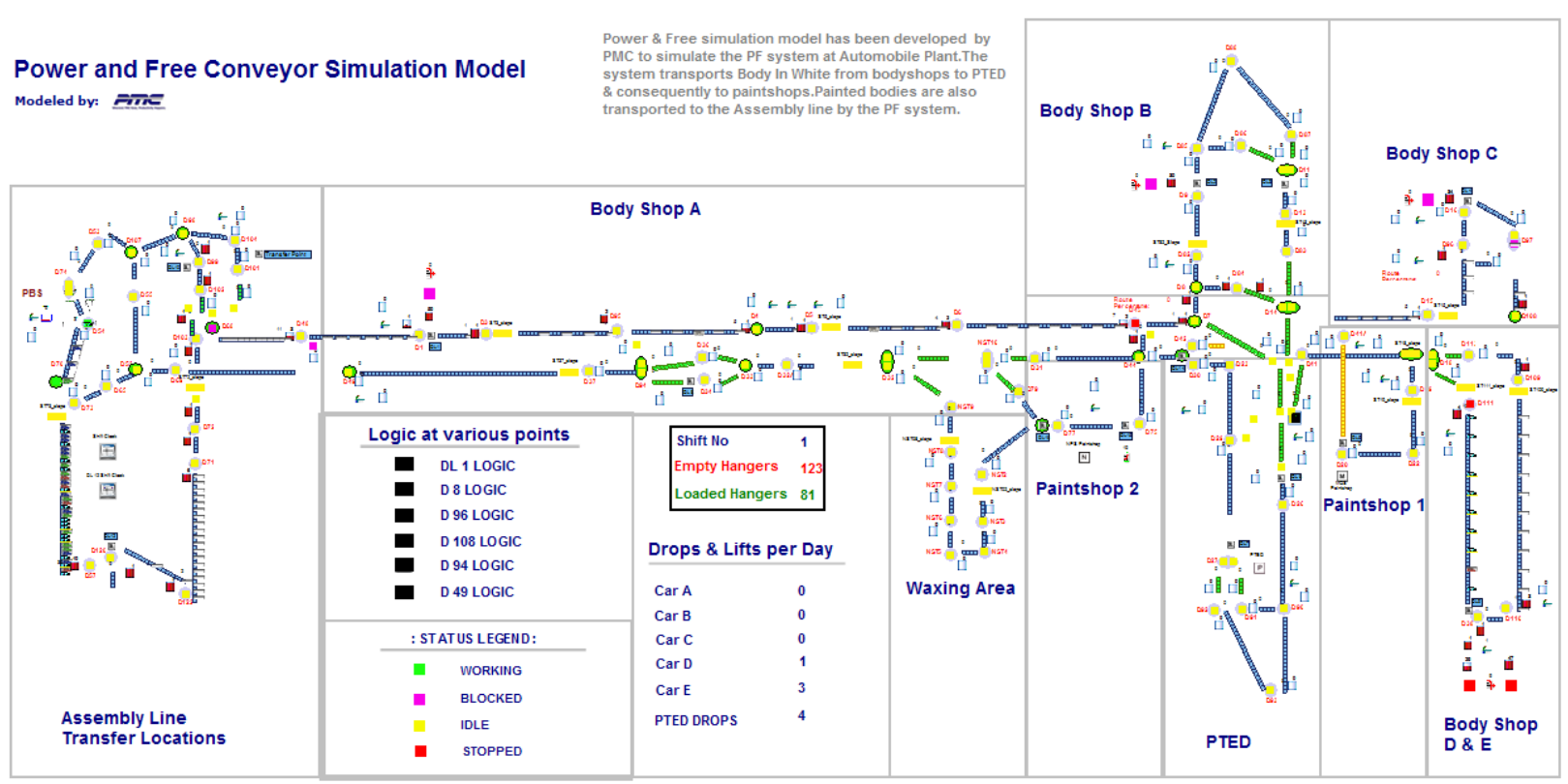

Figure 5: Model screenshot

Model verification is performed using techniques such as visual screening, dry runs, code checks, break points; trace files and tracking single entities through the model. Also, verification is performed using standard methodologies on both on individual building blocks as well integrated model comprising repeated module elements.

The model is validated by comparing model output parameters with those recorded in the system over the three previous months. Parameters used for validation include hanger utilization, body shop production levels and buffer contents. Several validation meetings were organized to ensure that model trends and patterns matched patterns that the system experts were used to measuring. The base line model was confirmed as validated at a throughput level of 635 drops per day at PTED.

\section{ANALYSIS AND RESULTS}

The simulation model verified that the power and free system could meet the current production demands. However, meeting the new target production levels would require significant changes to the way the system was being run. Several changes to decision rules were also proposed that would enable the plant to keep the P\&F system running in a delicate balance while avoiding deadlock occurrences.

Several output parameters were recorded and written out to MS Excel® to allow detailed performance analysis. The base simulation model throughput averaged out at 635 drops at PTED. Analysis of 


\section{Devikar, Garge, Vasudevan, Welekar and Williams}

this result was done by using following Time in State charts (TIS) and activity charts (samples shown in Figure 8 and Figure 9). The time in state charts for the base model indicate that there is considerable blockage at lift 12 which serves the assembly line. Since filled hangers get emptied at lift 12 and these empty hangers move to lift 1 to pickup bodies, blockage at lift 12 would cause a loss on the vehicle assembly line as well as loss in pickup of BIWs at the body shops. Blockage at lift 2 was a result of accumulation of empty hangers between lift 1 and lift 12. This in turn was result of piling of hangers between lift 1 and lift 6 cascading it up to lift 7. Similarly lift 7 was blocked because of accumulation of hangers till lift 15, which was a result of piling of hangers back till lift 12. This indicates an imbalance in the current system which can be attributed to excess hangers. This logical bottleneck analysis leads to the discovery of a closed loop bottleneck. Figure 9 marks this closed loops system and Figure 10 shows the logical analysis in a flowchart.

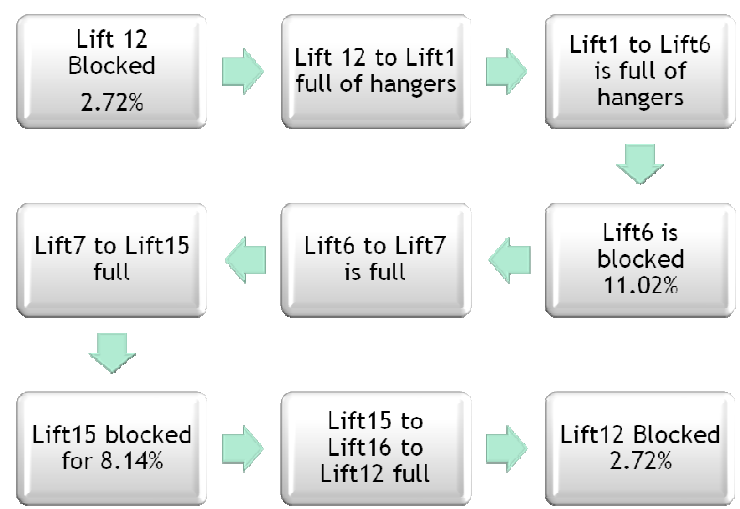

Figure 6: Logical analysis of blocking/congestion

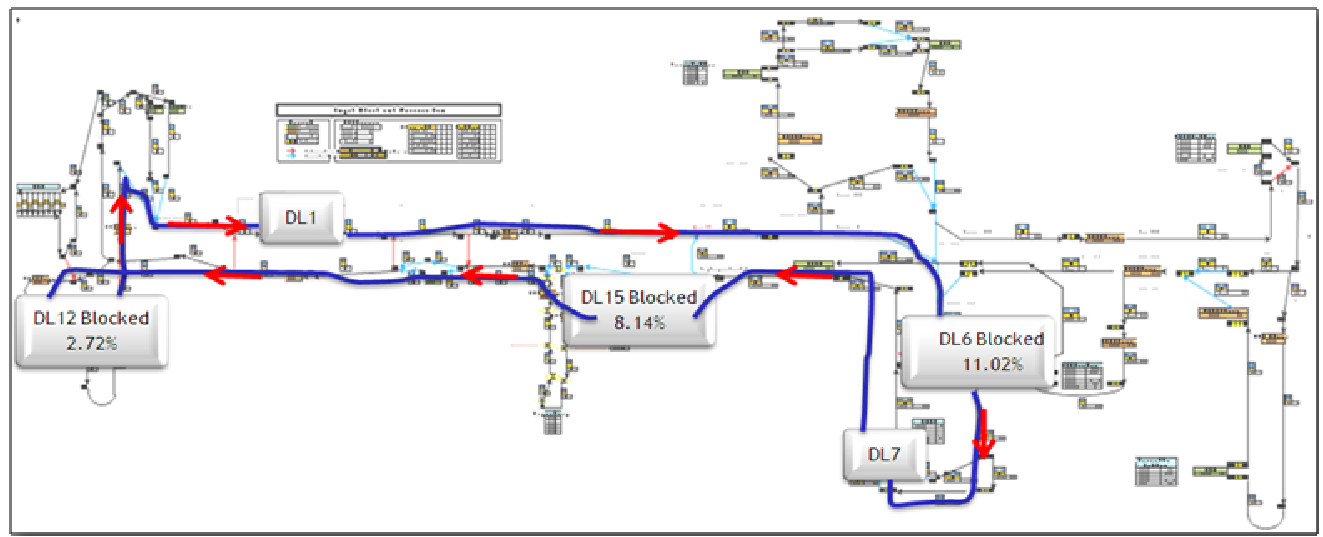

Figure 7: Analysis of Blockage Percentages Leads to Discovering a Bottleneck loop

Several experiments were conducted through a half factorial design and by considering investment constraints to try and improve system throughput to target levels. Figure 8 shows scenario results that summarize the throughput improvement roadmap. The model confirmed that by reducing number of hangers in the system, throughput climbs significantly. The sweet spot for number of hangers is 208 (which is a drop of 7 hangers from original levels). Dropping 7 hangers improves activities at PTED by 11 drops per day. Increasing body shop storage in steps increases PTED drops to the target levels -652 per day. 
Devikar, Garge, Vasudevan, Welekar and Williams

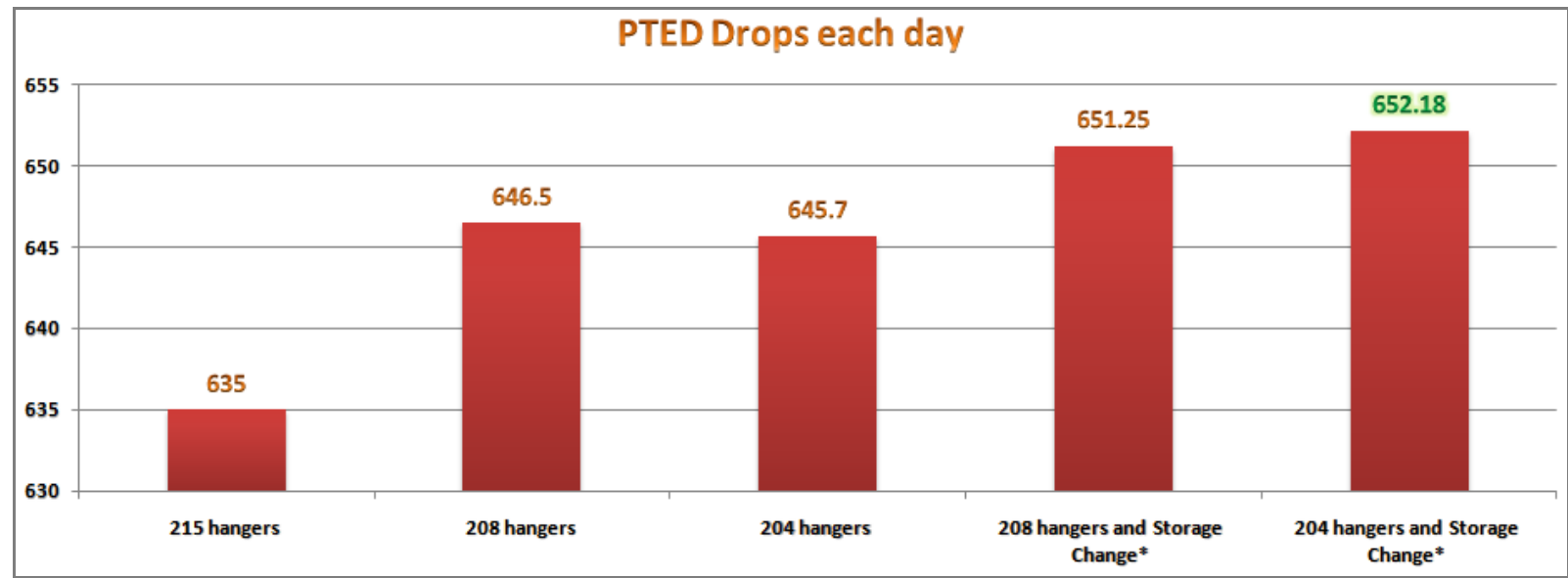

Figure 8: Scenario comparison summary chart

Figure 9 shows the number of pick-ups and drops completed at each drop lift in the system (stacked bar represents activity in individual shifts). This chart helps us validate the performance requirements of the power and free system. The chart shows that the model with recommended changes is able to meet the target of 652 drops at PTED. Studying the variation in PTED drops over time in each shift (Figure 10) helped the plant optimize PTED resources and balance production. The PTED is a key system because it links the critical body shops with the paint shops that are the design bottleneck in the plant.

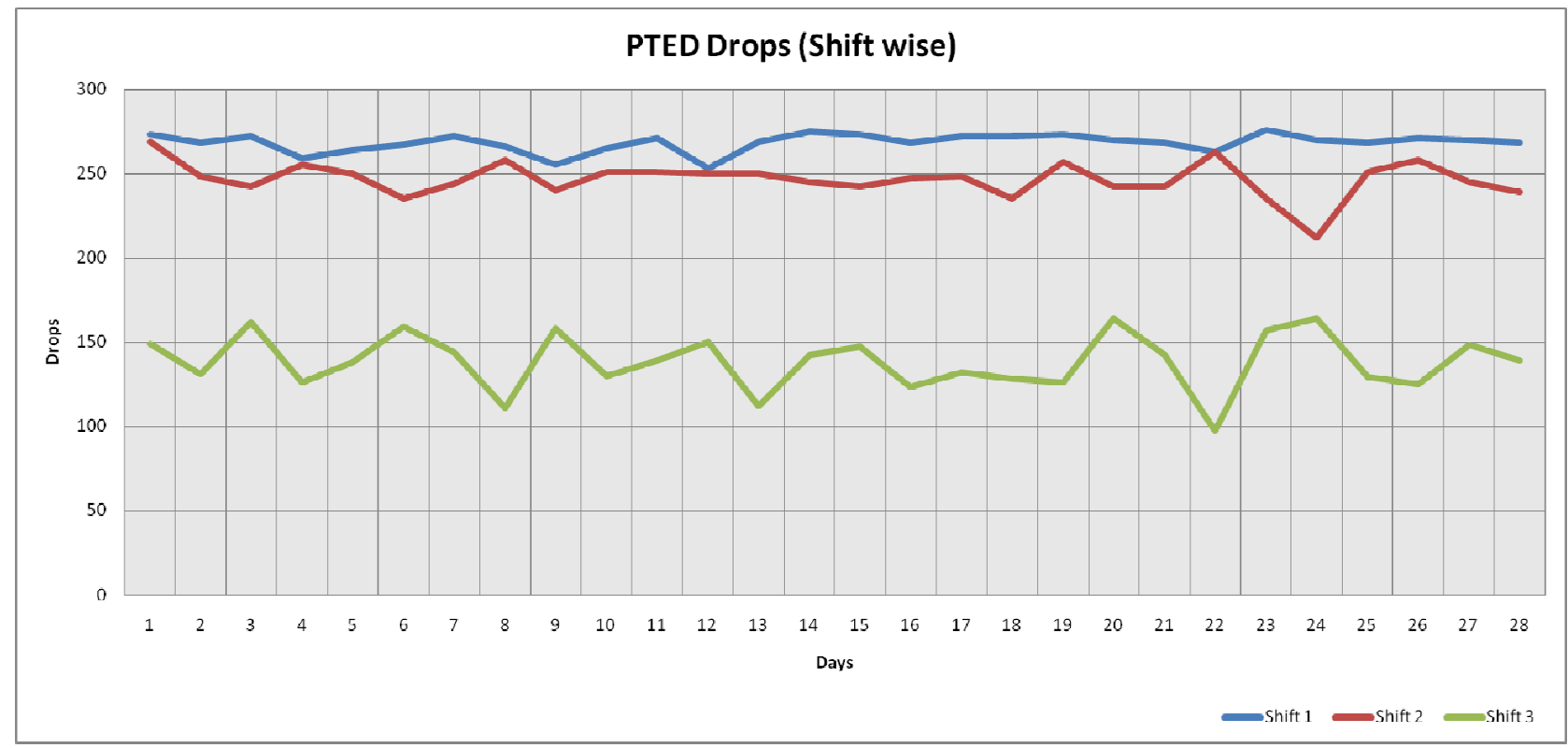

Figure 9: Shift wise PTED deliveries chart 


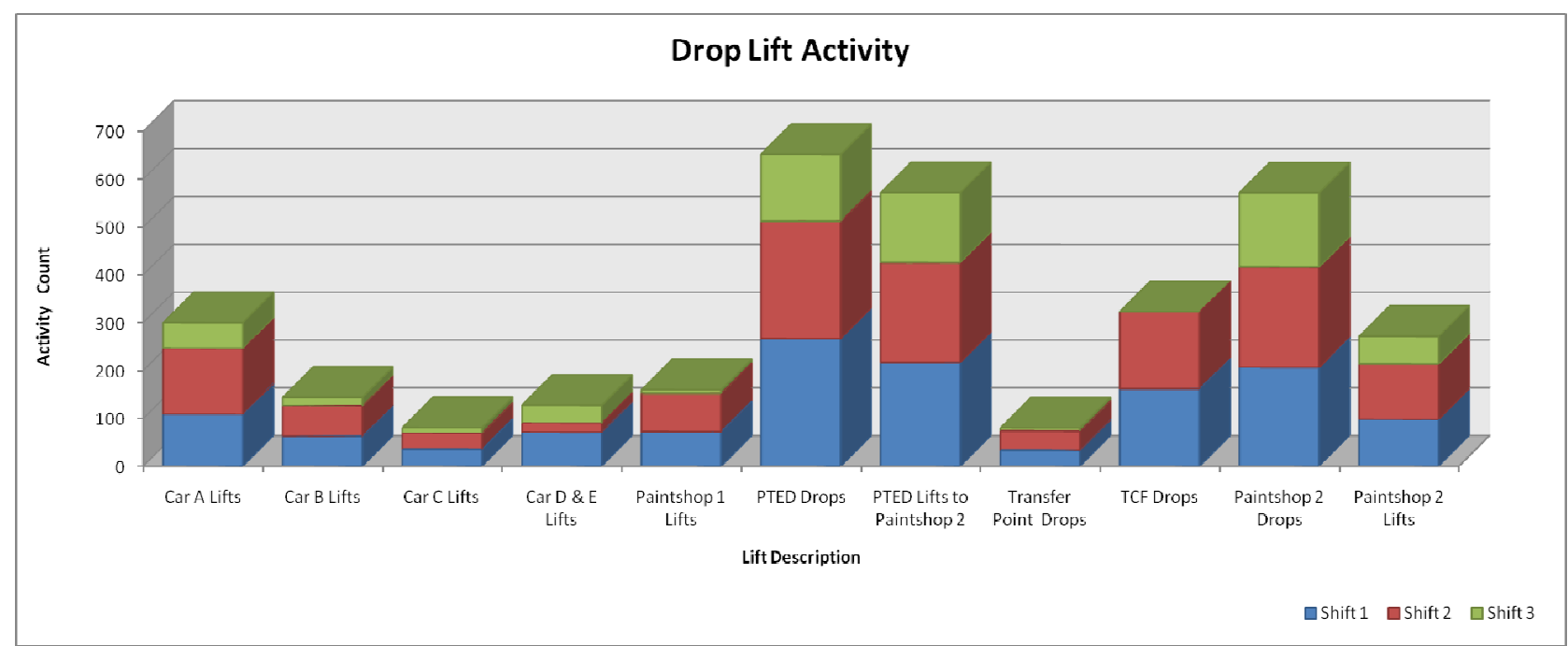

Figure 10: Drop lift activity chart

Figure 11 shows the time series accumulation of car bodies in the bodyshop buffer. Quantifying this accumulation pattern allowed the plant to size the storage system in such a way that body shop blockage could be minimized or eliminated. One of the throughput improvement action items to achieve target throughput involved expanding the sizes of two body shop buffers to reduce blockage and hence minimize hanger imbalances on the P\&F system. A graph of full and empty hangers on the power and free system is also plotted (Figure 12) and helps quantify the variation and steady state numbers.

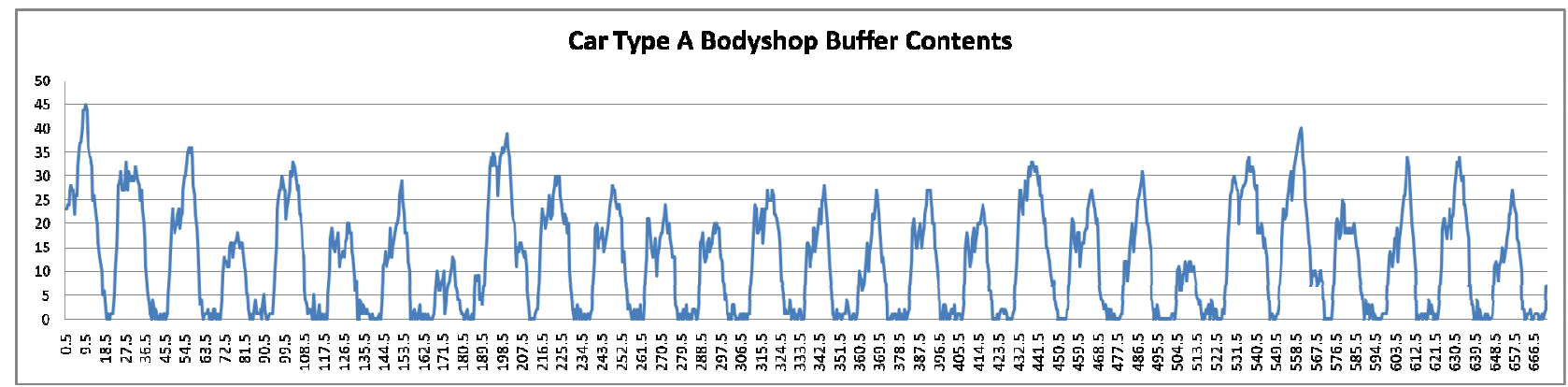

Figure 11: Bodyshop buffer time series graph

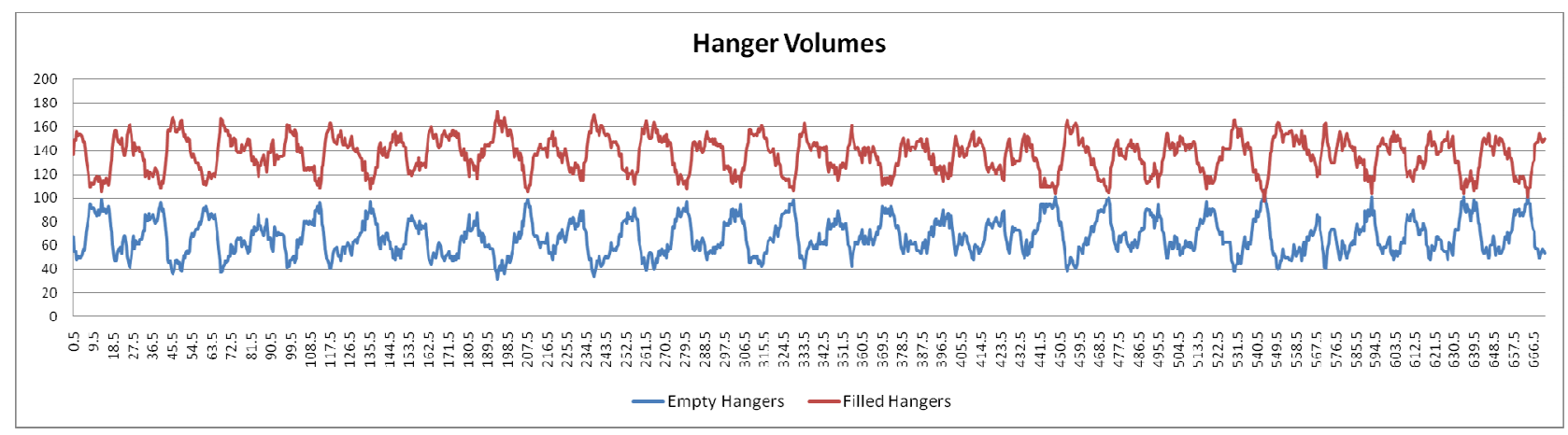

Figure 12: Time series chart showing hanger volume distribution 


\section{CONCLUSION}

A complex power and free system that transports multiple car body types across production shops within a flexible manufacturing plant is simulated and analyzed. The analysis is used to calendarize action items to increase production from present day levels to a future target level. The bottleneck analysis reveals a congestion pattern in a critical closed loop system. Reducing number of hangers in the system reduces congestion and betters system performance. This counter-intuitive recommendation was the most important and highest impact finding of this simulation project. Several other key performance indicators were also presented to help understand and improve delivery performance in several parts of the power and free system. Decision rules and routing policies were also revised based on iterative experimentation and analysis. The project team is now exploring options to replicate this model for analyzing P\&F performance in several other manufacturing plants across the country.

\section{REFERENCES}

Kambiz, F., and A. Balasubramanian. 2002. Solving Logistics and Transportation Problems in a Job Shop. In Proceedings of the 2002 Winter Simulation Conference ed. E. Yücesan, C. H. Chen, J. L. Snowdon, J. M. Charnes, 1052-1059. Piscataway, New Jersey: Institute of Electrical and Electronics Engineers, Inc.

Finch, B. J. 2008. Operations Now: Supply Chain Profitability and Performance, $3^{\text {rd }}$ edition. Boston, Massachusetts: The McGraw-Hill Companies, Incorporated.

Guasch, A., J. Figueras, and P. Fonseca. 2009. Factory Railway System. In Simulation-Based Case Studies in Logistics: Education and Applied Research, eds. Yuri Merkuryev, Galina Merkuryeva, Miquel Àngel Piera, and Antoni Guasch, 1-18.

Heragu, S. S., G. Meng, and H. Zijm. 2008. Stochastic Models for Facilities Logistics. In Facility Logistics, ed. Maher Lahmar. New York, New York: Auerbach Publications, 127-152.

Hoff, A., H. Vogelsang, U. Brinkschulte, and O. Hammerschmidt. 1997. Simulation and Visualization of Automated Guided Vehicle Systems in a Real Production Environment. In Proceedings of the $9^{\text {th }}$ European Simulation Symposium, eds. Winfried Hahn and Axel Lehmann, 391-395.

Jing, G., W. Gang, D. Kelton, J. C. Arantes, and A. A. Houshmand. 1998. Modeling a Controlled Conveyor Network with Merging Configuration. In Proceedings of the 1998 Winter Simulation Conference, eds. D. J. Medeiros, E. F. Watson, J. S. Carson and M. S. Manivannan, 1041-1048. Piscataway, New Jersey: Institute of Electrical and Electronics Engineers, Inc.

Miller, S., and D. Pegden. 2000. Introduction to Manufacturing Simulation. In Proceedings of the 2000 Winter Simulation Conference, eds. J. A. Joines, R. R. Barton, K. Kang, and P. A. Fishwick, 6366. Piscataway, New Jersey: Institute of Electrical and Electronics Engineers, Inc.

Vasudevan, K., R. Lote, E. Williams and O. Ulgen, 2009. High Speed Bottle Manufacturing Lines: Case Studies and Simulation Software Selection Techniques. In Proceedings of the 2009 Winter Simulation Conference, eds. M. D. Rossetti, R. R. Hill, B. Johansson, A. Dunkin and R. G. Ingalls. Piscataway, New Jersey: Institute of Electrical and Electronics Engineers, Inc.

\section{AUTHOR BIOGRAPHIES}

ASHISH DEVIKAR is an Industrial Engineer at Production Modeling India (PMI) in Nagpur, India. He has Bachelors in Industrial Engineering and a Post Graduate Diploma in Packaging Science. He has also served as TPM Secretariat in Ispat Steel Industries Pvt. Ltd., Nagpur, India. He has also undergone Six Sigma Green Belt training. He has worked on various simulation projects for manufacturing as well as service industries and has experience in Lean implementation. His email address is <adevikarapmcorp.com>. 


\section{Devikar, Garge, Vasudevan, Welekar and Williams}

NIKHIL GARGE is an Applications Engineer at Production Modeling India (PMI) in Nagpur, India. He has a Bachelor of Technology degree in Mechanical Engineering from Visveswaraya National Institute of Technology, Nagpur, India. His area of interest is applying discrete event simulation tools for manufacturing industries and warehouse facilities. His email ID is <ngarge@pmcorp.com $>$.

KARTHIK VASUDEVAN is a Technical Manager at Production Modeling Corporation (PMC) in Redmond, WA. His technical skills span simulation, software, controls and scheduling domains with experience in seven simulation software besides scheduling tools and programming languages. He received a Master's degree in Industrial Engineering from the University of Arizona, Tucson, USA and a Bachelor's degree in Electrical and Electronics Engineering from Sathyabama University, Chennai, India. He received the "Dean's award for Outstanding Graduate Student" and the "Award for Excellence" at the University of Arizona. He is an ASQ Certified Six Sigma Black Belt and APICS Certified in Production and Inventory management. At PMC, he has worked on several consulting projects spanning automotive, semiconductor, oil \& gas, steel, packaging, nuclear, warehousing, healthcare and service industries. He is an active Rotarian, serves on the organizing committee of the Winter Simulation Conference 2010, is a steering committee member of the Michigan Simulation Users Group and an officer in the Puget Sound Chapter of the IIE. His email ID is <kvasudevanepmcorp. com $>$.

RAJESH WELEKAR is the Director of Production Modeling India (PMI) in Nagpur, India. He has over 15 years of experience in the automobile industry. Rajesh has hands on experience in PMTS (predetermined motion time system) and has successfully completed a number of projects in the domain. He is also an expert in productivity improvement through wage settlement. Rajesh has earned a Bachelor's degree in Mechanical Engineering from Government college of Engineering from Karad in Maharashtra. He has also completed an Executive Program in Business Management from Indian Institute of Management - Calcutta. He is well versed and intersted in MIS, PMTS, Training on PMTS, Line Balancing, Layout Optimization and PMTS techniques. He can be reached at <rwelekar@pmcorp.com>.

EDWARD J. WILLIAMS holds bachelors and master's degrees in mathematics (Michigan State University, 1967; University of Wisconsin, 1968). From 1969 to 1971, he did statistical programming and analysis of biomedical data at Walter Reed Army Hospital, Washington, D.C. He joined Ford Motor Company in 1972, where he worked until retirement in December 2001 as a computer software analyst supporting statistical and simulation software. After retirement from Ford, he joined PMC, Dearborn, Michigan, as a senior simulation analyst. Also, since 1980, he has taught evening classes at the University of Michigan, including both undergraduate and graduate simulation classes using GPSS/H $\mathrm{H}^{\mathrm{TM}}$, SLAM $\mathrm{II}^{\mathrm{TM}}$, SIMAN ${ }^{\mathrm{TM}}$, ProModel ${ }^{\circledR}, \mathrm{SIMUL8}{ }^{\circledR}$, or Arena ${ }^{\circledR}$. He is a member of the Institute of Industrial Engineers [IIE], the Society for Computer Simulation International [SCS], and the Michigan Simulation Users' Group [MSUG]. He serves on the editorial board of the International Journal of Industrial Engineering Applications and Practice. During the last several years, he has given invited plenary addresses on simulation and statistics at conferences or seminars in Monterrey, México; İstanbul, Turkey; Genova, Italy; Rīga, Latvia; Göteborg, Sweden; and Jyväskylä, Finland. He has served as Program Chair of the 2004, 2005, and 2006 Summer Computer Simulation Conferences, and also for the 2005 IIE Simulation Conference. His university web page is: $\langle$ http://www-personal. umd.umich.edu/ williame $>$. 